\title{
HENRI MÉNUDIER
}

\section{ADENAUER, DE GAULLE UND DER ÉLYSÉE-VERTRAG NACH ALAIN PEYREFITTE}

Aus Anlaß des 40. Jahrestages des Élysée-Vertrages' ist es sehr nützlich, sich noch einmal sehr genau die Äußerungen von General de Gaulle anzuschauen, so wie sie Alain Peyrefitte niedergeschrieben hat, der mit de Gaulle zwischen 1959 und 1969 mehr als 300 Gespräche führte. Die getreue Niederschrift, bereichert durch zahlreiche Beobachtungen aus den Kabinettsitzungen oder anderen wichtigen Treffen, zeigt die Ausformung der Politik des Generals. In diesen Texten findet der Leser die Analyse der französischen und internationalen Probleme und seine manchmal recht hart formulierten Urteile über die bedeutendsten Persönlichkeiten dieser Epoche. Sehr viel weniger verklausuliert als bei den Diplomaten ist der Stil des Generals lebhaft und aufgeweckt, manchmal mit überraschenden Ausdrücken gewürzt.

1925 geboren, ehemaliger Schüler der École normale supérieur (ENS) und der École nationale d'administration (ENA) begann Alain Peyrefitte $(\dagger 1999)$ mit 22 Jahren seine diplomatische Karriere, in deren Verlauf er zu einem bekannten Spezialisten für China wurde. ${ }^{2} 1958$ zum Abgeordneten und Bürgermeister von Provins (Seine-et-Marne) gewählt - eine Stadt, in der er bis 1995 Bürgermeister bleiben wird -, machte er eine lange politische Karriere in den Reihen der Gaullisten. Er trat 1962 in die Regierung ein und blieb dort mit einigen Unterbrechungen bis 1981. Zu Zeiten von General de Gaulle war er nacheinander Informationsminister (1962-1966), Minister für wissenschaftliche Forschung und Atom- und Raumfahrt (1966-1967) und schließlich Minister furr nationale Erziehung (1967-68). Von 1962 bis 1966 war er außerdem noch Regierungssprecher und der Sprecher General de Gaulles. 1977 wurde er in die Académie française aufgenommen. ${ }^{3}$ Europe, Asnières 1993.

2

3 
Die Besonderheit des "Zeugnisses« von Alain Peyrefitte - der sich dagegen verwahrt, Memoiren ${ }^{4}$ geschrieben zu haben - besteht darin, nach 30 Jahren der Öffentlichkeit seine Aufzeichnungen aus der Zeit zugänglich zu machen, die er damals Tag für Tag niedergeschrieben hatte. Peyrefitte wollte also kein durch die Erinnerung aufgefrischtes Zeugnis ablegen, sondern eine wirkliches Dokument, eine "Quelle« aus erster Hand liefern, was die Zeitspanne von 30 Jahren vor der Publikation - die nach dem Gesetz von 1979 auch für die Konsultation der öffentlichen Archive gilt - als solche auch legitimieren sollte. Die typographische Form macht es dem Leser sofort möglich, zwischen der Niederschrift der noch ganz "heißen« Notizen und dem "Bindegewebe«, das der Autor zum Zeitpunkt der Vorbereitung der Veröffentlichung 1993-1994 den Beobachtungen hinzugefügt hat, zu unterscheiden. Die Bedeutung dieses Werkes liegt unter anderem darin, daß es die "Memoiren der Hoffnung " $"$ von de Gaulle vervollständigt, die aufgrund des Todes des Generals am 9. November 1970 unvollendet blieben, und die eine wwiederhergestellte« Erzählung für die Geschichte sind. ${ }^{6}$ De Gaulle hatte also nicht die Zeit, seine eigene Darstellung vom Élysée-Vertrag zu geben, weder vom französischen Weg zum Vertrag und noch von der enormen Enttäuschung, die auf die Hinzufügung der Präambel durch den Bundestag folgte, die das Dokument seines eigentlichen Sinnes beraubte.

Der Historiker muß deshalb auf andere Quellen zurückgreifen, um die Lükke zu füllen. Selbstverständlich sind die Erinnerungen von Peyrefitte dabei nicht das einzige Werk. Von den zahlreichen "Zeugnissen « über den General und über die deutsch-französischen Beziehungen in den 1960er Jahren ${ }^{7}$ wollen wir nur zwei nennen, in denen die Beziehung de Gaulles zu Deutschland im Zentrum der Darstellung steht: das Werk von Pierre Maillard, Germanist und

4 Alain PeYrefitTE, C'était de Gaulle, Bd. 1: „La France redevient la France«, Paris 2002, S. 9.

5 Charles DE GAulle, Mémoires d'espoir, Bd. 1: Le renouveau, 1958-1962; Bd. 2: L'effort, 1962-... (beim Tode des Generals waren nur zwei Kapitel für die Veröffentlichung fertiggestellt), Paris 1970/71.

6 Mit Bezug auf die »Mémoires d'espoir« kommentiert Jean-Louis Crémieux-Brilhac: „Die Mémoires d'espoir sind seine letzte Mission, wichtiger noch als das Regieren [...] In Wirklichkeit wollen die Mémoires d'espoir mehr noch als die Mémoires de guerre, ein Bouquet von Lektionen im Stile einer Erzählung sein. Sie sind im übrigen auch ein Testament. Und mehr noch als die Mémoires de guerre, die eine Lücke in den historischen Kenntnissen ausfüllten, war die getreue Nacherzählung der Ereignisse dem Bild, das von ihnen blieb, untergeordnet«; Einleitung zur Edition der Memoiren von de Gaulle in der Reihe Pléiade, Paris 2000, S. XLIX und LI.

7 Bezüglich der großen Unterschiede und dem Wert dieser Zeugnisse, vgl. Ulrich LAPPENKÜPER, Die deutsch-französischen Beziehungen 1949-1963. Von der "Erbfeindschaft« zur »Entente élémentaire«, München 2001, S. 23-24. 
ehemaliger französischer Botschafter, und das von Hermann Kusterer, dem Übersetzer von Bundeskanzler Konrad Adenauer. Pierre Maillard war von 1959 bis 1964 der diplomatische Berater des Generals, und er hat sein Werk nicht nur durch seine persönlichen Erinnerungen bereichern können, sondern greift auch auf die der anderen Mitarbeiter und Vertrauten von de Gaulle zurück, die er befragt hat. Gleichzeitig bemühte er sich - auch wenn er sich dagegen verwahrt - die Arbeit eines Historikers zu tun, so daß er den Weg in die Archive antrat. ${ }^{8}$ Das Euvre, das dabei entstand, ist eine minutiöse Arbeit, analytisch und interpretierend, mit zeitlichem Abstand geschrieben, viel mehr als nur eine unbearbeitete Erinnerung, sondem eher mit einer »Quelle» vergleichbar. Das Werk von Hermann Kusterer ist aufgrund der gewählten Methode dagegen eher ein Zeitzeugnis. ${ }^{9}$ Der Autor schreibt seine Erinnerungen nieder und behauptet nicht, im Besitz der absoluten Wahrheit zu sein (»Das ist meine Geschichte, und nur die meine ${ }^{10}$ ). Und Kusterer hat »die Pflicht zur Zurückhaltung«, von der Alain Peyrefitte spricht, offenbar noch weiter getrieben: Tatsächlich hat der Übersetzer des Bundeskanzlers regelmäßig seine Arbeitsnotizen vernichtet, sobald eine Mission beendet war. Um die Veröffentlichung seines Werkes vorzubereiten, hat er auf die Memoiren von de Gaulle und Adenauer, ${ }^{11}$ auf Publikationen von Originaldokumenten ${ }^{12}$ und auf eine sehr beschränkte Zahl von direkten Zeugenaussagen (Maurice Couve de Murville, ${ }^{13}$ Pierre Maillard, ${ }^{14}$ Horst Osterheld ${ }^{15}$ ) zurückgegriffen und dabei bewußt auf die Benutzung von sogenannter "Sekundärliteratur « verzichtet. Es handelt sich also sicherlich um ein Zeugnis, das 30 Jahre nach den Ereignissen wieder rekonstruiert wurde. Man sieht dagegen bereits in der Gegenüberstellung die Besonderheit der Arbeit des Chronisten Alain Peyrefitte: Nach einem Zeitraum, den ihm die »Zurückhaltung» auferlegt hatte, galt es seine unmittelbaren Zeugenaussage zu rekonstruieren, die er noch unter dem direkten Eindruck der Ereignisse niedergeschrieben hatte. Deshalb waren die Kommentatoren, als

8

9

10

"Konrad AdenaUER, Erinnerungen, 4 Bde., Stuttgart 1965-1968; DE GAULLE, (Anm. 5).

12 Konrad Adenauer, Briefe 1949-1951 und Briefe 1951-1953, Berlin 1985/1987; DERS., Teegespräche 1950-1954, Teegespräche 1955-1958, Teegespräche 1959-1961, Teegespräche 1961-1963, Berlin 1984-1992; Charles DE GAULLE, Lettres, Notes et Camets, 12 Bde., Paris 1980-1988; Ders., Discours et Messages, 5 Bde., Paris 1970.

13 Maurice Couve DE MURVILLE, Une politique étrangère, 1958-1969, Paris 1973.

14 Vgl. Maillard (Anm. 8), S. 14-15.

15 Horst OSTERFELD, Konrad Adenauer - Ein Charakterbild, Bonn 1973; DERS., »Ich gehe nicht leichten Herzens... « - Adenauers letzte Kanzlerjahre. Ein dokumentarischer Bericht, Mainz 1986; DERS., Außenpolitik unter Bundeskanzler Ludwig Erhard, 1963-1966, Düsseldorf 1992 . 
"C'était de Gaulle« erschien, so begeistert von der Lebendigkeit dieser Worte, die General de Gaulle wieder auferstehen ließen. ${ }^{16}$

Es ist deshalb völlig legitim, diese direkte Zeugenaussage eines »Getreuen« des Generals heranzuziehen, um zu versuchen, die Einstellung de Gaulles zur Bundesrepublik zum Zeitpunkt der Unterzeichnung des Élysée-Vertrages nachzuvollziehen. Die folgende Studie beschäftigt sich daher insbesondere mit den Reisen Adenauers nach Frankreich und de Gaulles nach Deutschland im Juli und September 1962, mit den Erwartungen, die de Gaulle in den Vertrag vom 22. Januar setzte und mit den Enttäuschungen (begrenzt unter Adenauer, heftig unter Erhard), die folgen sollten. Seine Meinung über den Zustand der Bundesrepublik und über die Politiker macht deutlich, was beinahe zum anfänglichen Scheitern des Vertrages geführt hätte und beleuchtet in einem grellen Licht den Wandel der deutsch-französischen Beziehungen während der Vertragsverhandlungen und als Folge des Vertrages. ${ }^{17}$

\section{Die Bewunderung de Gaulles für Adenauer}

Nach Ende des Algerienkrieges im Frühjahr 1962, dem definitiven Scheitern der Fouchet-Pläne im April ${ }^{18}$ und der britischen Unentschiedenheit in bezug auf Europa (erneut bestätigt bei dem Treffen zwischen Macmillan und General de Gaulle in Champs-sur-Marne am 2. und 3. Juni ${ }^{19}$ ), hatte der General nur noch Augen für Adenauer. Im vorausgehenden Jahr (vom 20. bis 23. Juni 1961) hatte er den Präsidenten der Bundesrepublik, den Christdemokraten Heinrich Lübke, ${ }^{20} \mathrm{zu}$ einem offiziellen Besuch empfangen, aber er hatte in ihm nur einen "Kaminaufsatz gesehen, der nicht zählt « ${ }^{21}$. Bundeskanzler Adenauer hingegen verkörperte für ihn Deutschland: Also beschloß er, alle Register zu

${ }^{16}$ Der Journalist Patrick Jarreau schrieb in Le Monde vom 21.10.1994: "Die Überraschung raubt einem den Atem. Man öffnet mit viel Vorfreude das Buch von Alain Peyrefitte über die Äußerungen, die Charles de Gaulle vor dreißig und mehr Jahren ganz privat gemacht hatte [...]. Nichts Veraltetes oder Verrostetes in diesen Worten [...]. Dreißig Jahre später erzwingen sie so viel Aufmerksamkeit, als würde er heute sprechen«.

17 Die Zitate stammen aus dem Buch von Alain PEYREFITTE, C'était de Gaulle, Paris 2002, 1960 Seiten (ein Wiederabdruck der drei Bände, die von den Verlagen De Fallois und Fayard zwischen 1994 und 2000 veröffentlicht wurden).

$18 \mathrm{Vgl}$. den Band Du Plan Fouchet au Traité franco-allemand de janvier 1963, in: Revue d'Allemagne et des pays de langue allemande, 29 (1997), 2.

19 Vgl. Documents Diplomatiques français (DDF) 1962, Bd. 21, Dokument 172, S. $554 \mathrm{ff}$.

20 Vgl. DDF 1961, Bd. 19, Dokument 300, S. 928ff.

21 Peyrefitte (Anm. 17), S. 167. 
ziehen, um so mehr, als nach dem Besuch des Kanzlers in Frankreich im Juli 1962 der General seinerseits Anfang September auch nach Deutschland reisen sollte.

De Gaulle erklärte gegenüber Alain Peyrefitte, daß Adenauer vor 1933 der Oberbürgermeister von Köln gewesen sei, sich während der Nazi-Zeit würdig verhalten habe und Deutschland seit 1949 mit Strenge regiere: ${ }^{22}$ "Niemand kann besser als er meine Hand ergreifen. Aber niemand kann sie ihm besser reichen als ich «.. ${ }^{23}$ Da der General während des Krieges ein unerbittlicher Gegner der Deutschen gewesen war, glaubte er: "Von mir erwarten sie Absolution für ihre Kriegsverbrechen«.

De Gaulle bewunderte auch die Tatsache, da es Adenauer innerhalb weniger Jahre gelungen war, der Bundesrepublik wieder einen besonderen Platz auf der internationalen Bühne verschafft zu haben, trotz der Einschränkungen, die nach wie vor auf ihr lasteten. Die Annäherung mit Frankreich hatte ihm sehr genützt; der General erinnerte bei mehreren Gelegenheiten daran, daß Westdeutschland wieder Besitz über seine Kohle und seinen Stahl erlangt hatte, die Saar wieder deutsch geworden sei und daß es wieder eine Armee aufbauen durfte. Ohne de Gaulle hätte es von dem gemeinsamen Markt sogar noch mehr profitiert, der zu Anfang nur die Landwirtschaft umfaßte. Adenauer what eingesteckt, was er konnte ${ }^{24}$. De Gaulle bedauerte jedoch, daß Adenauer bereits 1961 seinen Rückzug aus dem Kanzleramt zwei Jahre später angekündigt hatte: "Sobald man ankündigt, daß man gehen wird, regiert man nicht mehr ${ }^{25}$

Dennoch machte ihn die Bewunderung für den großen Mann nicht blind, und de Gaulle zeigte sich sehr kritisch gegenüber der Lage und der Entwicklung Deutschlands. Er urteilte, daß die deutsche Verfassung, "von den Engländern und Amerikanern gemacht«, den Nachteil habe, den Parteien zuviel Macht einzuräumen, weil sie kein Referendum zulasse, ${ }^{26}$ so daß das Volk, obwohl es sich über das Fernsehen gut informieren könne, im Abseits bleibe. Auch die Angriffe der deutschen Presse waren ihm ein Dorn im Auge, doch suchte er die Schuld dafür woanders: "Sie steht unter der Fuchtel der Angelsachsen ${ }^{27}$. Für de Gaulle war die florierende Wirtschaft kein Ersatz dafür, daß das Land seine moralischen Grundlagen noch nicht wieder gefunden hatte. Seine Einwohner würden unter der Kollektivschuld leiden, die Vernichtung

22 Vgl. Hans-Peter SCHWARZ, Adenauer. Der Aufstieg: 1876-1952, Stuttgart 1986; DERS., Adenauer. Der Staatsmann: 1952-1967, Stuttgart 1991.

23 PEYREFITTE (Anm. 17), S. 167.

24 Ibid. S. 900.

25 Ibid.

26 Ibid. S. 175.

27 Ibid. S. 812. 
fürchten und noch immer einem Vergangenheitskult pflegen. Zudem beobachtete er die ständige Abhängigkeit Deutschlands mit großem Mißtrauen: „Früher von den Preußen. Gestern von den Österreichern. Heute von den Amerikanern einerseits und den Russen andererseits ${ }^{28}{ }^{28}$

De Gaulle mochte das supranationale Europa nicht, und er fand sehr harte Worte für Jean Monnet, Robert Schuman und die Männer der IV. Republik, die seine Befürworter gewesen waren. Er hielt sie für "Kranke«, "bemitleidenswerte Persönlichkeiten«, vor allem darum bemüht, »den Vereinigten Staaten zu dienen «. Das supranationale Europa und die NATO bedeuteten ihm nicht viel und dienten auch zu nichts: "All das ist nichts, nichts, nichts ${ }^{29}$. Demgegenüber war ihm nicht entgangen, daß dieses supranationale Europa der Bundesrepublik um so besser gefiel, eben wegen seiner Abhängigkeit. De Gaulle hingegen sprach sich klar und deutlich für ein Europa der Regierungen und der Staaten aus, für ein sehr viel lockeres atlantisches System, win dem Europa auf gleicher Ebene mit den Amerikanern stünde, während diese gegenwärtig über alle Macht verfügten «. ${ }^{30}$

Die Besonderheit Deutschlands lag fur den General darin, daß es durch seine Teilung und durch die Suche nach einer eventuellen Wiedervereinigung sehr behindert werde. Er fand, da $B$ »diese Trennung nicht natürlich ist, sie war viel zu brutal, gegen die Natur der Dinge ${ }^{31}{ }^{31}$ Jeder Teil Deutschlands leide unter dieser Trennung, »eines Tages wird es sich dagegen auflehnen«. Es liege im egoistischen Interesse Frankreichs, möglichst lange ein geteiltes Deutschland aufrechtzuerhalten, aber diese Situation würde laut de Gaulle nicht ewig andauern: "Deutschland wird wiedervereinigt werden ${ }^{32}$. Er beobachtete, wie Adenauer die Westintegration der Bundesrepublik einer Wiedervereinigung vorzog, zeigte sich selber aber bisweilen ungeduldiger als der Kanzler und manchmal sogar visionär, indem er die Deutschen ermutigte, wieder eine Nation zu werden. Er glaubte, daß die Deutschen in Frankreich ein Modell sähen.

Frankreich wisse, wohin es gehe, Deutschland hingegen wisse es nicht. Das Volk "fühlt sich nicht wirklich geführt. Es lebt unter Institutionen, denen es sich kaum verbunden fühlt. Die Regierung ist gespalten ${ }^{33}{ }^{33}$ Sicherlich gebe es „die außergewöhnliche Persönlichkeit des Bundeskanzlers«, aber er würde schon bald von der Macht abtreten.

33 Ibid. S. 177. 


\section{Adenauer in Frankreich, de Gaulle in Deutschland}

Adenauer wurde in Frankreich vom 2. bis 9. Juli 1962 wie ein Staatschef empfangen ${ }^{34}$ und zeigte sich sehr "gerührt" von der außergewöhnlichen Mühe, die der General aufgewandt hatte, um diesen Besuch persönlich zu organisieren. ${ }^{35}$ Dieser endete mit einem symbolischen Akt, einem Defilée französischer und deutscher Truppen in Mourmelon, an der Marne (wo sich Franzosen und Deutsche während der letzten beiden Weltkriege gegenübergestanden hatten), und mit einer Messe in der Kathedrale von Reims, einer im Ersten Weltkrieg schwer heimgesuchten Stadt, in der am 8. Mai 1945 die deutsche Kapitulation unterzeichnet worden war. Bevor sie sich trennten, hatte de Gaulle gegenüber Adenauer noch einmal betont, daß der Besuch wein sehr bedeutender Akt und ein großer Erfolg ${ }^{36}$ gewesen sei, die Herzlichkeit sei aufs Tiefste vom französischen Volk empfunden worden. Alain Peyrefitte notiert dennoch, daß die Öffentlichkeit eher spärlich vertreten war und es auch Zeichen der Feindseligkeit gegeben hatte!

Die Zurückhaltung der Franzosen gegenüber dem Bundeskanzler unterschied sich deutlich vom Triumph des Generals während seiner offiziellen Visite in Deutschland vom 4. bis 9. September 1962. ${ }^{37}$ Der General hielt alle seiner 14 Reden in Deutsch - die Texte wurden in Französisch verfaßt, dann übersetzt und schließlich auswendig gelernt. Während der Kabinettsitzung am 12. September im Élysée sprach der Außenminister, Maurice Couve de Murville, von weiner Begeisterung, wie es sie in der Bundesrepublik noch nie gegeben habe, ja vielleicht nicht einmal in der Welt. Es ist das Fernsehen, das diesen Schneeballeffekt erzielt hat $\ll$. De Gaulle, der den französischen Widerstand gegen Hitler verkörperte, war den Deutschen sofort ans Herz gewachsen, indem er sich in ihrer eigenen Sprache an sie wandte. Er war die geeignetste Person, um ihnen zu verzeihen: "Er hat dies ohne Anbiederei getan, indem er sie gleichwertig behandelte, wie Partner, die zu Freunden geworden sind und rehabilitiert vor der Geschichte«. ${ }^{38}$

De Gaulle gestand selbst ein, daß diese Reise ein "historischer Akt ${ }^{39}$ war. Die zahlreichen direkten Kontakte mit den Deutschen hatten ihm die Notwendigkeit einer praktischen Zusammenarbeit gezeigt, »ein enormes Bedürfnis nach Annäherung der Jugend [...]. Wir werden Vorschläge machen«. Man

34 Vgl. DDF 1962, Bd. 22, Dok. 4, S. 9 ff.

35 PEYREFITTE (Anm. 17), S. 169.

36 Ibid. S. 171.

37 Vgl. DDF 1962, Bd. 22, Dok. 67, S. 172ff.

38 PEYREFITTE (Anm. 17), S. 176.

39 Ibid. S. 177. 
sollte nicht vergessen, daß die Äußerung eines solchen Wunsches in einem politisch sehr angespannten Klima getätigt wurde. Nach dem Attentat von Petit-Clamart (22. August 1962) und dem Mißtrauensantrag gegen die Regierung Pompidou am 5. Oktober standen in Frankreich im Oktober und November das Referendum über die Direktwahl des Präsidenten und vorgezogene Neuwahlen an. Von Oktober bis Dezember sollte die Bundesrepublik durch die "Spiegel-Affäre« aufgerüttelt werden, die Adenauer zwang, sein Kabinett umzubilden, und ihn weiter schwächte. Hinzu kamen die Kuba-Krise in der zweiten Oktoberhälfte, die spärlichen Ergebnisse der Gespräche zwischen de Gaulle und Macmillan am 15. und 16. Dezember ${ }^{40}$ und die anglo-amerikanische Übereinkunft von Nassau über den Atomteststop, Ereignisse, die de Gaulle und Adenauer dazu veranlaßten, ihre Zusammenarbeit weiter zu intensivieren. ${ }^{41}$

\section{Ein schlecht aufgenommener »großartiger Akt»}

Die Situation im Jahre 1963 hatte natürlich auch Einfluß auf die Debatte über den Élysée-Vertrag. Am 14. Januar sprach sich General de Gaulle gegen den Beitritt Großbritanniens in die Europäische Wirtschaftsgemeinschaft (EWG) aus und weigerte sich, an der multilateralen Atomstreitmacht teilzunehmen, die die Vereinigten Staaten vorgeschlagen hatten, ${ }^{42}$ im Juni zog er die französischen Flottenverbände im Ärmelkanal und im Atlantik aus der NATO zurück. ${ }^{43}$ John F. Kennedy - am 22. November in Dallas erschossen - wurde von Lyndon B. Johnson abgelöst, der über die internationalen Verhandlungen nur ungenügend auf dem Laufenden war. Die deutsche Politik ihrerseits war durch den Rücktritt von Adenauer schwer getroffen, dem am 16. Oktober Ludwig Erhard als Bundeskanzler folgte. Am 15. Juli hielt Egon Bahr, Berater des Re-

${ }^{40}$ Vgl. DDF 1962, Bd. 22, Dok. 200, S. 535ff.

41 Vgl. Jacques BARIÉTY, De Gaulle, Adenauer et la genèse du traité de l'Élysée du 22 janvier 1963, in: Institut Charles de Gaulle (Hg.), De Gaulle en son siècle, Paris 1992, S. 352-364; im selben Werk siehe auch den Beitrag von Hans-Peter SCHWARZ, Le président de Gaulle, le chancelier fédéral Adenauer et la genèse du traité de l'Élysée, S. 364 373; vgl. auch Henri MÉNUDIER u.a. (Hg.), La RFA et la construction de l'Europe (19491963), Paris 1999.

42 Charles DE GAULLE, Discours et Messages, Bd. 4: Pour l'effort: août 1962 - décembre 1965, Paris 1970, S. $61 \mathrm{ff}$.

${ }^{43} \mathrm{Vgl}$. DDF 1963, Bd. 23, Dok. 218, S. 640ff. Siehe dazu auch die Reaktion in der BRD, ibid. Dok. 217, S. $637 \mathrm{ff}$. 
gierenden Bürgermeisters von Berlin, Willy Brandt, seine berühmte Rede in Tutzing zum »Wandel durch Annäherung«, die Ankündigung der neuen Ostpolitik. $^{44}$

De Gaulle akzeptierte, daß das deutsch-französische Abkommen, das nach seiner Reise nach Deutschland vorgeschlagen worden war, nicht nur ein Protokoll, sondern ein richtiger Vertrag in aller Form werden sollte und damit der Ratifizierung beider Parlamente bedurfte. Adenauer fürchtete, da $B$ ein einfaches Abkommen keine Bindekraft für seinen Nachfolger haben könne. Der General, der das Scheitern der Fouchet-Pläne im April 1962 nicht vergessen hatte, verkündete also am 3. Januar 1963 im Kabinett: "Wir werden zu zweit tun, woran uns die Belgier und die Holländer gehindert haben. Aber diese beiden zählen sehr viel mehr als alle vier zusammen ${ }^{45}$ Nach der Unterzeichnung des Vertrages am 22. Januar und zur Überraschung der anwesenden Gäste umarmte de Gaulle Adenauer; Alain Peyrefitte notierte, daß die beiden hauptsächlich Beteiligten woffensichtlich selbst nicht damit gerechnet ${ }^{46}$ hatten. Premierminister Georges Pompidou wurde von dieser »Gefuihlswallung « überrascht. Für de Gaulle war Deutschland bis dahin vor allem ein Synonym für Krieg gewesen: "Und auf einmal kehrte sich in diesem Moment der einzigartigen Brüderlichkeit alles um«, vermerkte Alain Peyrefitte. ${ }^{47}$

Adenauer und de Gaulle unterstrichen sofort die Bedeutung des Vertrages. Für den Bundeskanzler bedeutete er die "Krönung seines Lebens «, ${ }^{48}$ für den französischen Präsidenten handelte es sich um einen "großartigen Akt ${ }^{49}{ }^{49} \mathrm{Er}$ erläuterte am 23. Januar, daß die deutsch-französische Annäherung eine grundlegende Realität sei, die fortdauern müsse: Es könne keine europäische Zusammenarbeit ohne eine deutsch-französische Zusammenarbeit geben, man müsse die Sachen zusammen in Angriff nehmen: "All das sollte sehr große Auswirkungen haben ${ }^{50}{ }^{50} \mathrm{Im}$ Verlauf einer offiziellen Reise in das Departement Charente lobte er am 14. Juni 1963 die deutsch-französische Kooperation, die auf wimmenses Unglück» folge. Als er über Frankreich und Deutschland sprach, fügte er hinzu: "Wenn man genau nachdenkt, haben sie eine Revolution vollzogen und ein herrliches Beispiel gegeben«. Er meinte

4

Vgl. Heinrich POTTHOFF, Im Schatten der Mauer. Deutschlandpolitik 1961 bis 1990 , Berlin 1990, S. 31ff.; Daniel KüCHENMEISTER, Detlef NAKATH (Hg.), Architekt und Brückenbauer. Gedanken Ostdeutscher zum 80. Geburtstag von Egon Bahr, Bonn 2002.

45 PEYREFITTE (Anm. 17), S. 814.

46 Tbid. S. 815.

47 Ibid. S. 816.

48 Ibid.

49 Ibid.

so Ibid. S. 817. 
sogar, daß Frankreich »eine der größten Taten in seiner Geschichte« vollbracht habe. $^{51}$

Im Buch von Alain Peyrefitte finden sich wenige Kommentare über den Inhalt des Vertrages. Für den militärischen Sektor hielt der General fest, daß der Bundeskanzler sich mehr und mehr beunruhige, denn er zweifele daran, daß die Vereinigten Staaten sein Land im Falle eines sowjetischen Angriffs auch mit Atomwaffen schützen würden, so daß das Projekt einer multilateralen Atomstreitmacht Deutschland nur sehr begrenzten Nutzen bringe. Auf kulturellem Sektor hoffte de Gaulle, daß der Vertrag die einen wie die anderen zum Erlernen der Sprache des Partnerlandes anregen würde. Auf die Bemerkung Georges Pompidous, der deutsche Föderalismus werde die kulturelle Zusammenarbeit nicht erleichtern, antwortete de Gaulle: „Deutschland ist föderal und das ist auch viel besser so", denn er wünschte kein wzentralisiertes Reich auf der anderen Seite ${ }^{52}$ Der sehr kleine Bereich, der der Wirtschaft im Vertrag eingeräumt wurde, erklärte sich für Paris aus dem Wunsch Adenauers, den Vorsitzenden der Europäischen Kommission, Walter Hallstein, nicht zu verletzen. De Gaulle glaubte vor allem, daß Adenauer vor allem nicht Ludwig Erhard nach Paris mitnehmen wollte, dessen geringe Begeisterung für Paris er kannte. Und er fugte als Kenner hinzu: „Er ist ein alter Fuchs«. ${ }^{53}$

Das negative internationale Echo, das der Vertrag auslöste, interessierte de Gaulle wenig. Für ihn war es das wichtigste, mit Adenauer einer Meinung gewesen zu sein in der Weigerung, die EWG auf Großbritannien auszuweiten. So konnten die Angelsachsen nicht die "Intimität zwischen den Galliern und den Germanen« stören. Einige Kommentare in der Presse betonten die Gefahr eines deutsch-französischen Übergewichts in Europa, andere fragten sich, ob das Abkommen nicht nur toter Buchstabe bleiben würde. De Gaulle wollte sich nicht vom "Erdbeben « beeindrucken lassen, das er ausgelöst hatte: »Man darf sich nicht darüber wundern, daß Eifersüchteleien hervorbrechen. Es ist besser, Neid auszulösen als Mitleid «. ${ }^{54}$

Die Sowjets ihrerseits fürchteten nicht ohne Berechtigung, daß der ÉlyséeVertrag in einer atomaren deutsch-französischen Zusammenarbeit enden würde (auch wenn dies bereits im Juni $1958^{\text {s5 }}$ von de Gaulle ausgeschlossen worden war) und die Bundesrepublik die Westmächte in einen Krieg gegen Moskau hineinziehen könnte. Auch hier blieb de Gaulle stahlhart: "Die Sowjets wedeln mit der deutschen Gefahr um einen Vorhang aus Rauch zu er-

Ibid. S. 819 .

53 Ibid. S. 818.

54 Ibid. S. 819.

s5 Vgl. Georges-Henri SoUTOU, L'alliance incertaine. Les rapports politico-stratégiques franco-allemands, 1954-1996, Paris 1996, S. 136ff. 
richten ${ }^{56}$ Ganz im Gegenteil hielt er Deutschland nicht für expansiv und glaubte, daß sein nun noch engeres Band mit Frankreich es für die UdSSR ungefährlicher machte. Im Kabinett schloß er am 6. Februar 1963: "Von West nach Ost, die ganze Welt protestiert. Also sind wir auf dem richtigen Weg: Alles läuft zum besten «. ${ }^{57}$

IV. Die Präambel - „Sie benehmen sich wie Schweine«

Schon am Tag nach der Unterzeichnung des Élysée-Vertrages und aufgrund der vom General am 14. Januar 1963 vertretenen Position gegen Großbritannien und gegen die multilaterale Streitmacht, fragte sich die französische Diplomatie, ob der Bundestag den Vertrag ratifizieren und Adenauers Nachfolger ihn respektieren würde. De Gaulle rief am 23. Januar in Erinnerung, da $\beta$ Deutschland die Initiative zum Vertrag ergriffen habe, und daß, wenn es ihn nicht ratifiziere, "wir eine andere Orientierung finden werden ${ }^{58}$ Er zog allerdings auch die Schwächung Adenauers in Betracht: "Wir müssen vermeiden, den Deutschen lästig zu werden [...]. Aber sie müssen auf jeden Fall verstehen, daß, wenn sie den Vertrag nicht ratifizieren, es ein für alle Mal Schluß ist. Dann werden wir die Orientierung ändern «. ${ }^{59}$ Am 20 . Februar befragte Alain Peyrefitte den General erneut über die Möglichkeit einer NichtRatifizierung durch den Bundestag. Dieser antwortete, daß er nicht sehr an diese Möglichkeit glaube, aber daß, wenn es sein müsse, er sich über das Fernsehen erneut direkt an das deutsche Volk wenden werde, und daß dies "einen durchschlagenden Erfolg" haben werde. Er fügte allerdings hinzu: „Behalten Sie das auf jeden Fall für sich «. ${ }^{60}$

Unter dem Druck der Amerikaner und unterstïtzt von Jean Monnet nahm der Bundestag schließlich am 16. Mai 1963 fast einstimmig eine Präambel an. Indem diese die multilateralen Verpflichtungen der Bundesrepublik hervorhob, reduzierte sie die Bedeutung des Vertrages. Frankreich ließ offiziell verlauten, daß es dagegen nichts einzuwenden habe, aber in Wirklichkeit ereiferte sich General de Gaulle sehr heftig gegen die Amerikaner und die deutschen Abgeordneten, indem er erneut die Drohung eines Allianzenwechsels anklin-

Ibid. S. 383.

Ibid. S. 818.

s9 Ibid. S. 820 .

60 Ibid. S. 823. 
gen ließ: "Die Amerikaner versuchen unseren Vertrag seines Inhalts zu berauben. Sie wollen daraus eine leere Hülle machen. Warum all das? Weil die deutschen Politiker Angst haben, vor den Angelsachsen nicht genügend auf dem Bauch zu kriechen! Sie benehmen sich wie Schweine. Sie würden es verdienen, daß wir den Vertrag kündigen und einen Allianzenwechsel vollziehen und uns mit den Russen einigen! « ${ }^{61}$

\section{Der Vertrag, die jungen Mädchen und die Rosen}

Als der Vertrag schließlich ratifiziert wurde, stimmte de Gaulle einem Treffen am 4. und 5. Juli 1963 mit Adenauer in Bonn zu. ${ }^{62}$ Am 3. Juli zeigte er sich über die Präambel und über das Funktionieren der deutsch-französischen Kooperation enttäuscht, "die nicht so effizient ist, wie ich sie mir vorgestellt hatte ${ }^{63}{ }^{63} \mathrm{Am}$ Abend des gleichen Tages empfing er die französischen Parlamentarier im Élysée-Palast, die sich skeptisch gegenüber dem Bestand des Vertrages zeigten. Der General antwortete mit seiner berühmt gewordenen Parabel: "Verträge sind wie junge Mädchen und Rosen: Das hält solange wie es hält. Wenn der deutsch-französische Vertrag keine Anwendung fände, wäre er nicht der erste in der Geschichte«. Am 5. Juli fügte Konrad Adenauer in Bonn hinzu, daß die Rosen, vor allem die mit Dornen, sehr widerstandsfähig seien: "Diese Freundschaft zwischen Frankreich und Deutschland ist wie ein Rosenstock, der immer Knospen und Blüten tragen wird«. Der General schloß sich dieser Ansicht an: "Sie haben recht, Herr Bundeskanzler, unser Vertrag ist keine Rose, auch kein Rosenstock, sondern ein Rosengarten. Eine Rose hält nur einen Vormittag lang. Aber ein Rosengarten unbegrenzt, wenn man sich die Mühe macht, ihn zu pflegen . $^{64}$

Trotz seiner Enttäuschung über die Präambel erkannte der General sofort den Nutzen der deutsch-französischen Gipfeltreffen. Obwohl von jedem Treffen keine Wunder zu erwarten seien, vermutete er jedoch, daß mneue Gewohnheiten" schnell »enge Bande" schaffen könnten: "Aber es ist eine kindische und boshafte Einstellung, jedes Mal eine Erwartungshaltung zu schaffen, die nur enttäuscht werden kann «. ${ }^{65}$

a) Ibid.

62 Vgl. DDF 1963, Bd. 24, Dok. 6, S. 14ff; AAPD 1963II, Dok. 216-219, S. 689ff.

${ }^{63}$ Peyrefitte (Anm. 17), S. 826.

64 Ibid. S. 827

o5 Ibid. 
Das letzte offizielle Treffen zwischen de Gaulle und Adenauer fand am 21. und 22. September $1963^{66}$ (der Bundeskanzler trat am 16. Oktober von seinem Amt zurück) im Schloß von Rambouillet statt. Es gab Gelegenheit zu einem großen Tour d'horizon durch die internationale Politik (insbesondere über die Rolle der UdSSR und Chinas), über die Atlantische Allianz und über Europa. De Gaulle wiederholte seine Skepsis gegenüber der multilateralen Streitmacht, in der er nur ein Schimäre für Deutschland sah. Adenauer hingegen brachte seine völlige Übereinstimmung mit der Atompolitik Frankreichs zum Ausdruck. Trotzdem dominierte beim General die Enttäuschung: »In der letzten Zeit war unsere Zusammenarbeit nicht sehr erfolgreich. [...] Die militärischen Fragen zwischen Frankreich und Deutschland gehen nicht gut voran « ${ }^{67} \mathrm{De}$ Gaulle zufolge sei der Beitritt Bonns zum Abkommen vom 5. August 1963 über das Verbot von Kernwaffenversuchen in der Luft, im Weltraum und unter Wasser, das von den Vereinigten Staaten, Großbritannien und der Sowjetunion unterzeichnet worden war, überflüssig, da Deutschland bereits erklärt habe, keine atomare Bewaffnung anzustreben: "Diese Meinungsverschiedenheit zwischen Frankreich und Deutschland ist aufmerksam beobachtet worden«, betonte er. ${ }^{68}$

De Gaulle bedauerte, daß der Élysée-Vertrag noch nicht über das Stadium einer Absichtserklärung hinausgekommen sei, und das müsse sich in Zukunft ändern. Er wiederholte sein Glaubensbekenntnis: „Es gibt keine andere europäische Wirklichkeit als Deutschland und Frankreich, und, wenn diese beiden Realitäten nicht auf allen Domänen vereint sind, werden wir untergehen « ${ }^{69}$. Mit dem Blick in die Vergangenheit zog Adenauer seinerseits eine Bilanz seiner Zeit im Kanzleramt: "Die deutsch-französische Freundschaft ist das wichtigste Ergebnis meiner Amtszeit während dieser 14 Jahre, in denen ich die deutsche Politik geleitet habe $\ll{ }^{70}$ Beim Apéritif vertraute er den Gästen sogar an: „Und die persönliche Freundschaft, die wir unter uns geknüpft haben, Herr General, ist eine der wenigen Freuden, die mein politisches Leben begleitet haben «."

Die beiden Staatsmänner waren voneinander fasziniert. Nach der Abfahrt Adenauers aus Rambouillet vertraute der General seiner Umgebung an: "Dieser Mann ist ein Wunder. Mit 88 Jahren noch diese geistige Schärfe, diese

6o Vgl. DDF 1963, Bd. 24, Dok. 103, S. 283ff; AAPD 1963II, Dok. 354-357, S. 1185 ff.

67 PeyrefitTe (Anm. 17), S. 831

68 Ibid.

${ }^{69}$ Tbid. S. 832.

70 Ibid.

${ }^{71}$ Ibid 
Präsenz zu haben und über 14 Jahre ohne Kräfteeinbußen regiert zu haben, das ist zweifellos ein einzigartiges Phänomen in der Geschichte «. ${ }^{72}$

\section{Ludwig Erhard - »ein Trottel«}

Der neue Kanzler kam am 21. November 1963 nach Paris. ${ }^{73}$ Vor diesem Treffen glaubte der General, daß Ludwig Erhard die privilegierte Allianz mit Frankreich nicht brechen und mit voranschreiten werde, denn das deutsche Volk wolle den großen Schwung der Aussöhnung nicht bremsen. Frankreich werde ihn nicht auffordern, sich zwischen ihm und den Vereinigten Staaten zu entscheiden. Für de Gaulle war Deutschland ein beschütztes Land, »der deutschen Nation das Rückrat gebrochen worden«, während die französische Nation immer noch "aufrecht und fest auf ihren beiden Beinen « stehe. ${ }^{74}$

Während des Treffens in Paris vertraute der Sprecher der Bundesregierung, Günther von Hase, Alain Peyrefitte an, daß Adenauer vielleicht freiwillig in den Schatten des General getreten sei, wohingegen Erhard sich als "Gleicher unter Gleichen « verstand. ${ }^{75}$ Dennoch äußerte sich der General recht positiv über diesen ersten Gipfel: «Das deutsch-französische Koordinatensystem ist solider als es aussieht. Erhard kann sich, auch wenn er es will, nicht von ihm lösen, aber ich glaube, er will es gar nicht. Er bemüht sich offensichtlich zu gefallen $\ll .{ }^{76}$ Hingegen war das Urteil über den Bundesaußenminister eindeutig: "Schröder ist der Mann der Angelsachsen. Er ist der typische falsche Zeuge; er hat nur ein Ziel, mir zu widersprechen «. ${ }^{77}$

Trotz der Verpflichtungen vom 21. und 22. November traten sehr bald Meinungsverschiedenheiten über die gemeinsame europäische Agrarpolitik auf. Am 11. Dezember urteilte de Gaulle, "dass es weder Fisch noch Fleisch sei ${ }^{78}$ und beklagte sich über das unangebrachte, um nicht zu sagen inakzeptable Verhalten der Bundesregierung ${ }^{79}$. Der Bundeskanzler scheine zu einem Ergebnis kommen zu wollen, aber sein Außenminister stelle sich ihm ständig in den Weg.

72 Ibid. S. 840.

73 DDF 1963, Bd. 24, Dok. 207, S. 532ff; AAPD 1963III, Dok. 421-423, S. 1455 ff.

74 PeYRefitTE (Anm. 17), S. 842.

75 Ibid. S. 843.

76 Ibid. S. 845.

77 Ibid.

78 Ibid. S. 846.

79 Ibid. S. 847. 
Im Frühjahr 1964 störten weitere europäische Fragen die deutsch-französischen Beziehungen. De Gaulle kündigte gegenüber Alain Peyrefitte an, $\mathrm{da} B$ er »die Deutschen anbrüllen« werde [...]. Ich werde Erhard die kalte Schulter zeigen ${ }^{80}{ }^{80}$ Er warf dem Kanzler vor, den Vertrag in einen "Schwindel « zu verwandeln. Streitpunkt blieb das Verhältnis zu den USA. Vor dem Beginn des Gipfeltreffens mit Ludwig Erhard am 3. Juli 1964 in Bonn ${ }^{81}$ traf de Gaulle Adenauer im Bundestag. Er, der doch wegen seiner geradezu militärischen Pünktlichkeit gefürchtet war, zog die Unterhaltung mehr in die Länge als vorgesehen, so da $B$ er Ludwig Erhard warten ließ, der darüber verärgert war. Als Entschuldigung sagte er ihm: "Ich war mit Konrad Adenauer zusammen. Ich war bezaubert und gefangen. Ich habe die Zeit nicht vorübergehen sehen «. ${ }^{82}$

In Paris, am 7. Juli 1964, während einer Unterhaltung mit Alain Peyrefitte nannte de Gaulle Erhard einen "Trottel«, der sich „mit aller Welt gut versteht ${ }^{83}{ }^{83}$ Während der Pressekonferenz am 23. Juli bemühte sich der General nicht mehr, seinen Streit mit dem Bundeskanzler zu verbergen, als er seine Bilanz über den Vertrag zog: "Man muß sehr wohl feststellen, auch wenn der deutsch-französische Vertrag in einigen Bereichen Teilergebnisse gebracht hat, auch wenn er die beiden Regierungen und ihre Administrationen zu zahlreichen Kontakten veranlaßt hat, die wir unsererseits alles in allem für recht nützlich halten und die auf jeden Fall sehr angenehm sind, so ist dennoch bis jetzt keine gemeinsame Linie zu erkennen ${ }^{84}{ }^{84}$

Am 12. November sagte er, da $\beta$ $\backsim$ Deutschland nicht wisse, was es wolle. Es weiß nicht, was in seinem Kopf vorgeht [...]. Mitleiderregend«. Bonn und Washington schlossen einen Rüstungsvertrag ab, so da $\beta$ de Gaulle Bonn umgehend vorwarf, "sich den Amerikanern vollends zu Füßen zu werfen « und den Élysée-Vertrag und Europa zu verraten. ${ }^{85}$ Das Zerwürfnis mit Bonn ließ den General sogar den Dialog mit der UdSSR und den Ländern des Ostblocks suchen, so wie er es im Dezember 1944 mit Stalin in Moskau getan hatte. Dennoch, in der deutschen Frage blieb er bei seinem Standpunkt. Er weigerte sich, die DDR anzuerkennen, denn »die Regierung von Pankow ist im Ausland gebildet worden«. Und fast schon visionär fuhr er fort: "Sie würden nicht eine Sekunde überleben, wenn die Sowjets abhauen; so wird es im übrigen auch enden. Dies ist eine absolut künstliche Regierung ${ }^{86}{ }^{86}$ De Gaulle erklärte dann,

${ }^{80}$ Ibid. S. 853.

${ }^{81}$ Vgl. DDF 1964, Bd. 26, Dok. 6, S. 1553; AAPD 1964II, Dok. 180-188, S. 713ff.

82 PeyrefitTe (Anm. 17), S. 854.

${ }^{83}$ Ibid. S. 859.

${ }^{84}$ De Gaulle (Anm. 42), S. 229.

85 PeYrefitTe (Anm. 17), S. 867.

${ }^{86}$ Tbid. S. 872. 
daß er das China Maos am 27. Januar 1964 anerkannt habe, weil die Regierung in Peking "von den Chinesen und niemand anderem " gebildet worden sei. Die Wiedervereinigung setze nicht nur die Zustimmung der Sowjets voraus, sondern auch die Anerkennung der Oder-Neiße-Grenze durch Bonn und dessen Verzicht auf Atomwaffen: "Sie müssen schon zugeben, die Deutschen, $\mathrm{da} ß$ sie besiegt worden sind und daß, wenn man besiegt worden ist, auch bezahlen muß ${ }^{87}{ }^{87}$ Dieses sagte de Gaulle Anfang Januar 1965!

Die negative Meinung des Generals sollte sich bestätigen. Der Gipfel vom 11. und 12. Juni 1965 in Bonn verlief eher schlecht. ${ }^{88}$ Auf dem Rückflug nach Paris erklärte der Präsident: DDie Deutschen sind erheblich geschwächt seit Adenauer nicht mehr da ist. Erhard ist kein Staatsmann. Er ist ohne Charakter ${ }^{89}{ }^{89}$

Der rasche Autoritätsverlust des Kanzlers führte am 27. Oktober 1966 zum Rückzug der liberalen Minister aus der Bundesregierung. Erhard trat am 30. November zurück: Er hatte nur drei Jahre regiert, während Adenauer 14 Jahre geblieben war. In der Kabinettsitzung, die am gleichen Tag in Paris abgehalten wurde, faßte de Gaulle seine Meinung so zusammen: „Erhard war unbeständig. Schröder war hartnäckig feindlich«. Das Urteil über die Große Koalition, die am 1. Dezember gebildet wurde, war positiv, wenn auch nuanciert: "Mit Kiesinger werden die Beziehungen einfach sein, und, wenigstens, was die Gefühle angeht, ist Brandt auch ganz anständig «. ${ }^{90}$ Es fand tatsächlich eine Annäherung statt, aber zwei Fragen sollten eine Verstimmung zwischen dem General und die Regierung Kiesinger auslösen: der Beitritt Großbritanniens in die EWG und das Ende des Prager Frühlings 1968.

Die französischen und deutschen Positionen in den wichtigsten Fragen der Außenpolitik lagen sehr viel weiter auseinander als während der Jahre unter Erhard. Die Meinungsverschiedenheiten über die Finanzierung der gemeinsamen Agrarpolitik führten auf französischer Seite zu einer Blockade der europäischen Institutionen (die Politik des »leeren Stuhls« vom 1. Juli 1965 bis 30. Januar 1966). Die Regierung Erhard unterstützte die amerikanische Politik in Vietnam (die massiven Bombardements eingeschlossen), während de Gaulle diese in seiner aufrüttelnden Rede in Phnom-Penh am 30. August 1966 verurteilte. ${ }^{91}$ Die multilaterale Streitmacht, an die die Regierung Erhard geglaubt hatte, scheiterte. Frankreich blieb weiterhin auf Distanz zu den Verei-

87 Ibid. S. 873.

88 AAPD 1965II, Dok. 242-249, S. 1002ff.

89 PeYREFITTE (Anm. 17), S. 884.

90 Ibid. S. 1403.

91 Vgl. Maurice VAÏSSE, La grandeur. Politique étrangère du général de Gaulle 1958-1969, Paris 1998, S. 532ff; Georges-Henri SoUTOU, La guerre de cinquante ans. Les relations Est-Ouest 1943-1990, Paris 2001, S. 470ff. 
nigten Staaten (Austritt aus der NATO im März 1966, die Reise de Gaulles in die UdSSR Ende Juni 1966), ${ }^{92}$ was sich auch nach dem ersten französischen Atomtest am 5. September nicht ändern sollte.

\section{Ein widerstandsfähiger Rosengarten}

Nach dem Scheitern der Fouchet-Pläne im April 1962, hatte de Gaulle zusammen mit der Bundesrepublik machen wollen, was mit dem Europa der Sechs gescheitert war; daher die Bedeutung, die er den Reisen in der zweiten Jahreshälfte 1962 beimaß und seine Erwartungen an den Élysée-Vertrag. Die Schwächung Adenauers, dann dessen Ablösung durch das Tandem Erhard/Schröder sowie die amerikanischen Feindseligkeiten mußten seinen Plan, ein europäisches Europa gegen ein amerikanisches Europa durchzusetzen, zunichte machen. Trotz dieser Enttäuschungen waren mit der feierlichen Besiegelung der deutsch-französischen Aussöhnung, der Einrichtung von Abstimmungsmechanismen zwischen Frankreich und der Bundesrepublik und der schüchternen Annäherung an die UdSSR und die Ostblockstaaten in diesen Jahren auch Lichtblicke zu erkennen gewesen. Diese und andere Entwicklungen lassen sich im Buch von Alain Peyrefitte nachlesen, der den deutschfranzösischen Regierungsalltag in grellem Licht darstellt, sehr weit entfernt vom gewundenen Stil der offiziellen Kommuniques. Das Wunder ist bis heute, $\mathrm{daß}$ der Élysée-Vertrag die starken Spannungen überdauert hat und somit immer mehr jenem widerstandsfähigen Rosengarten ähnelte, der von Charles de Gaulle und Konrad Adenauer angelegt worden war.

(Übersetzung: Birgit Schöne-Martens)

92 Vgl. Jacques BARIÉTY, Die deutsch-französische Annäherung. De Gaulles Reise nach Moskau im Jahre 1966 und die Deutsche Frage, in: Peter R. WEILEMANN, Hanns Jürgen KÜSTERS, Günter BUCHSTAB (Hg.), Macht und Zeitkritik. Festschrift für Hans-Peter Schwarz zum 65. Geburtstag, Paderborn 1999, S. 243-254. 
